\title{
The pNNx Heart Rate Variability Statistics: An Application to Neuroautonomic Dysfunction of Clozapine-Treated Subjects
}

\section{Jong-Hoon $\mathrm{Kim}^{1}$ \\ Sang Hoon $\mathrm{Yi}^{2}$ \\ Yong Min Ahn ${ }^{3,4}$ \\ Kyu Young Lee ${ }^{5}$ \\ Seung Ae Yang ${ }^{6}$ \\ Yong Sik Kim ${ }^{3,4}$}

${ }^{1}$ Department of Psychiatry,

Gil Medical Center, Gachon University

of Medicine \& Science, Incheon,

Institute of Basic Science \& School

of Computer Aided Science,

Inje University, Gimhae,

3Department of Psychiatry and

Behavioral Science,

Seoul National University

College of Medicine, Seoul,

IInstitute of Human

Behavioral Medicine,

Seoul National University, Seoul,

Department of Psychiatry,

Eulji University School of Medicine, Daejeon,

${ }^{6}$ Clinical Research Institute,

Seoul National University Hospital,

Seoul, Korea

\section{Correspondence \\ Yong Sik Kim, MD \\ Department of Psychiatry and \\ Behavioral Science, \\ Seoul National University \\ College of Medicine, \\ 101 Daehak-ro, Jongno-gu, \\ Seoul 110-744, Korea \\ Tel +82-2-2072-2204 \\ Fax +82-2-744-7241 \\ E-mail kys@snu.ac.kr}

(c) This is an Open Access article distributed under the terms of the Creative Commons Attribution Non-Commercial License (http://creativecommons.org/licenses/by-nc/3.0) which permits unrestricted non-commercial use, distribution, and reproduction in any medium, provided the original work is properly cited.
Objective The percentage of successive normal cardiac interbeat intervals greater than 50 msec (pNN50) is a widely used heart rate variability measure, which is useful in identifying the neuroautonomic dysfunction of psychiatric disorders. However, pNN50 is only one member of a larger family of $\mathrm{pNNx}$ statistics, where $\mathrm{x}$ is greater than $0 \mathrm{msec}$. The potential application of the general pNNx statistics has not yet been explored in the psychiatric field. The authors examined the pNNx statistics in clozapine-treated subjects and normal controls to evaluate the usefulness of the general $\mathrm{pNNx}$ statistics.

Methods Sixty-one schizophrenic patients treated with clozapine and fifty-nine normal controls were evaluated. Probability values for the differences between the groups at each pNN value (range: $\mathrm{pNN1-pNN100)}$ were calculated using data obtained from a 30-minute electrocardiogram.

Results The conventional pNN50 and pNNx values with $\mathrm{x}<50 \mathrm{msec}$ were all significantly lower in the patient group $(\mathrm{p}<0.05)$. The distinction between the two groups was more prominent at $\mathrm{pNN}$ values less than $50 \mathrm{msec}$ than that observed at pNN50. The maximum separation between groups occurred at pNN5 $\left(68.2 \pm 19.1\right.$ vs. $\left.22.5 \pm 20.5, \mathrm{p}<10^{-22}\right)$.

Conclusion The $\mathrm{pNNx}$ with $\mathrm{x}<50$ msec provided more robust discrimination between the groups than the conventional pNN50, suggesting the importance of analyzing very small variations of interbeat interval in discriminating normal and pathological heart rate patterns. The results also suggest that the general pNNx statistics may be applied and useful in evaluating the neuroautonomic dysfunction in patients treated with clozapine, complementing the traditionally computed pNN50 value.

Psychiatry Invest 2009;6:294-298

Key Words Heart rate variability, pNNx statistics, Schizophrenia, Clozapine, Neuroautonomic dysfunction.

Received: August 16, 2009 Revised: October 6, 2009 Accepted: October 27, 2009 Available online: November 9, 2009

\section{Introduction}

The analysis of heart rate variability (HRV), i.e., the variation in the cardiac interbeat interval over time, has proven to be useful in evaluating the neurocardiac function in various neuropsychiatric disorders and in patients receiving psychotropic medications. ${ }^{1-5}$ It is a useful non-invasive method providing a dynamic window into the alterations of complex neuronal activity in the central and peripheral nervous systems, and has significant utility in broader clinical and research applications. ${ }^{6}$

Recently, it has been demonstrated that linear and novel non-linear measures of HRV are different in patients with antipsychotic treatment compared to normal control subjects, suggesting significant neuroautonomic abnormalities associated with antipsychotics. $^{7-11}$

Among the various parameters of HRV, the pNN50 statistic, which is defined as the 
percentage of successive normal interbeat intervals greater than $50 \mathrm{msec}$, is one of the most widely used measures. $^{12,13}$ Previous reports have suggested that pNN50 was diminished in patients receiving antipsychotics such as clozapine or olanzapine. ${ }^{14,15}$ Considering that pNN50 reflects the vagal modulation, these results imply the neuroautonomic abnormality associated with decreased parasympathetic modulation in patients treated with these drugs. $8,10,14,15$

However, the pNN50 value is only one member of a larger family of pNNx statistics, where $\mathrm{x}$ is greater than 0 msec. ${ }^{16}$ Mietus et al. ${ }^{16}$ reported that $\mathrm{pNN}$ thresholds as low as $20 \mathrm{msec}$ or less were more useful and powerful than the standard $50 \mathrm{msec}$ threshold in discriminating normal subjects and pathological group such as heart disease, and suggested the utility of evaluating the general pNNx statistics rather than selecting only one fixed measure.

The applications of the general pNNx statistics and their usefulness have not yet been explored or rigorously tested in patients with antipsychotic treatment in which the HRV has already proven to be both practical and useful in evaluating the autonomic functioning., 4,17-19

In the present study, we comprehensively examined the pNNx statistics in normal controls and clozapine-treated patients to identify the optimum $\mathrm{pNN}$ values distinguishing the two groups and to evaluate the usefulness of the general $\mathrm{pNNx}$ statistics in patients treated with clozapine.

\section{Methods}

\section{Subjects}

The study protocol was approved by the Institutional Review Board of the Seoul National University Hospital, and all procedures used in the study were conducted in accordance with international ethical standards, Declaration of Helsinki. Sixty-one schizophrenic subjects (44 men, 17 women) treated at the Seoul National University Hospital Schizophrenia Outpatient Clinic, and 59 normal control subjects ( 34 men, 25 women) were evaluated. Informed consent was obtained from all subjects after a full explanation of the study procedure.

The patients were diagnosed according to the Diagnostic and Statistical Manual of Mental Disorders, 4th edition. ${ }^{20}$ None of the patients had suffered either from any diseases known to affect the autonomic cardiac function, such as cardiovascular, neurological or endocrinological diseases, or from drug dependence. The patients had a mean age of $34.0 \pm 7.8$ years and a mean duration of illness of $10.0 \pm 5.1$ years. Of 61 patients, 9 (14.8\%) patients were married, and $52(85.2 \%)$ were unmarried or divorced. The mean number of admissions in the past was 3.6 \pm 2.2 . The mean Clinical Global Impression-Se- verity $^{21}$ score was $2.9 \pm 0.6$ and the mean current Global Assessment of Functioning ${ }^{22}$ score was $54.9 \pm 14.5$. The mean total Positive and Negative Syndrome Scale ${ }^{23}$ score of the patients was $66.1 \pm 11.6$ (range $=41-88$ ).

All patients had been treated with stable doses of clozapine as monotherapy for at least 4 weeks. The mean daily dose of clozapine was $257.6 \pm 109.1 \mathrm{mg}$. The normal control subjects were screened by a complete medical and psychiatric examination and none had a history of any disease or medication that might affect the autonomic nervous system. There were no significant differences between the patient and control groups with regard to age (mean years: $34.0 \pm 7.8$ vs. $31.4 \pm 9.0, \mathrm{t}=1.64, \mathrm{p}=0.10$ ) or gender distribution (male \%: 72.1 vs. $57.6, \chi^{2}=2.77, \mathrm{p}=$ $0.10)$.

\section{Assessment of heart rate variability}

\section{Data Acquisition and Preprocessing}

To exclude the influence of diurnal variations, the HRV measurements were begun between 10:00 a.m. and 11:00 a.m. ${ }^{24}$ All subjects were instructed to avoid coffee and nicotine for at least one hour prior to the assessment of their autonomic function. After each subject had been allowed to adapt to the experimental conditions for about 15 minutes, a 30-minute single channel (3-lead) electrocardiogram (ECG) recording was performed in the seated position at complete rest. The ECG signal was amplified using an OP-Amplifier (MAX232) and was digitized at a sampling rate of $400 \mathrm{~Hz}$ (width path $0.05-35 \mathrm{~Hz}$ ). From the ECG of each subject, HRV was extracted by using previously proposed preprocessing procedures such as low-pass filtering, detrending, and R-peak detection of differential ECG signals. ${ }^{2,25}$ In the event of failure to extract HRV from the ECG due to noise, recordings were excluded. Recordings with non-sinus beats that were more than $1 \%$ of the total number of beats were also excluded. Premature beats and artifacts were carefully eliminated automatically and manually by visual inspection of all RR intervals.

\section{The pNNx Statistics}

The statistical time-domain measures of instantaneous heart rates are divided into two classes: 1) those derived from direct measurements of normal-to-normal intervals, and 2) those derived from the differences between successive interbeat intervals. One of the approaches to the measurement of HRV is via the percentage of absolute differences in successive normal sinus intervals that are greater than some threshold value. The most commonly used threshold is $50 \mathrm{msec}$, and the statistic obtained is termed pNN50. ${ }^{12}$ The conventional 50 msec used in the 
pNN50 measurements represents an arbitrary threshold, and is only one member of a general pNNx family of statistics. In the present study, the general $\mathrm{pNNx}$ statistics were obtained by estimating the percentage of successive RR interval differences whose absolute value exceeds $\mathrm{X}$ msec. ${ }^{16}$

\section{Statistical analysis}

Probability values for the differences between the groups at each pNN value were calculated using two-tailed t-tests as previously proposed. ${ }^{16}$ The interclass correlations between the HRV measures were assessed using Pearson's two-tailed correlation test. A critical value of $\mathrm{p}<0.01$ was used in statistical evaluation. Statistical analyses were performed using Statistical Package for the Social Sciences (SPSS) version 17.0 (SPSS, Chicago, IL, USA).

\section{Results}

A comparison of the $\mathrm{pNNx}$ measures between the groups is shown in Figure 1 and Table 1. The mean data length was not significantly different between the patient and control groups (1912.9 \pm 422.9 vs. $1868.0 \pm 365.6$, $\mathrm{t}=0.66, \mathrm{p}=0.51)$. The conventional $\mathrm{pNN} 50$ value and other pNNx parameters such as pNN10, pNN20, pNN30, and pNN40 were all significantly lower in the patient group than in the control group $(\mathrm{p}<0.05)$. However, as shown in Figure 1, the distinction between the two groups was more prominent at $\mathrm{pNN}$ values less than $50 \mathrm{msec}$ in comparison with that observed at the conventional pNN50 value. The maximum separation between groups occurred at pNN5 $\left(68.2 \pm 19.1\right.$ vs. $\left.22.5 \pm 20.5, \mathrm{p}<10^{-22}\right)$. In order to check the reliability of $\mathrm{pNNx}$, the interclass correlations between the square root of the mean squared differences of successive normal sinus intervals (RMSSD), the high frequency (HF) power and $\mathrm{pNNx}$ were examined. In the patient group, the $\mathrm{pNNx}$ values with $\mathrm{x} \leq 20 \mathrm{msec}$ were shown to correlate significantly well with RMSSD $(\mathrm{r}=0.99$ with $\mathrm{pNN} 10)$ and with $\mathrm{HF}(\mathrm{r}=0.96$ with $\mathrm{pNN} 20)$. In contrast to the patient group, the $\mathrm{pNNx}$ values with $\mathrm{x} \geq 30$ msec showed most significant correlations with RMSSD ( $r=0.97$ with $\mathrm{pNN} 30)$ and with HF ( $\mathrm{r}=0.94$ with pNN50) in the control group.

\section{Discussion}

In the present study, the pNNx statistics beyond the standard pNN50 were comprehensively examined to evaluate their usefulness in discriminating normal subjects and clozapine-treated patients. We found that $\mathrm{pNNx}$ with $\mathrm{x}<50$ msec provides more robust discrimination between the groups than the conventional pNN50 value. To the best
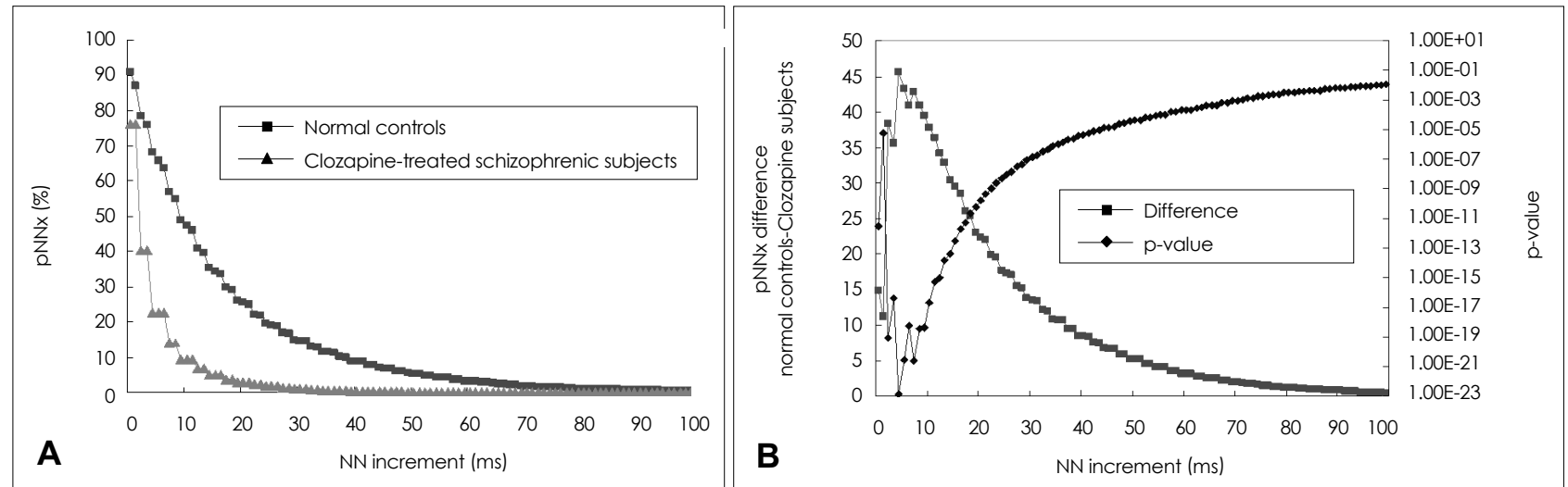

FIGURE 1. A: The percentage of successive normal cardiac interbeat intervals greater than each given interval (pNNx) versus the NN interval in normal control $(n=59)$ and clozapine-treated schizophrenia $(n=61)$ groups. B: The differences between the mean pNNx values of normal control subjects and those of schizophrenic patients and the corresponding $p$ values. The most significant separation between the two groups is around $x=5$ msec.

TABLE 1. Comparison of the pNNx measures between clozapine-treated schizophrenic patients and normal control subjects

\begin{tabular}{ccccc}
\hline pNNx measures & Patient group $(\mathrm{N}=61)$ & Control group $(\mathrm{N}=59)$ & t value & $\mathrm{p}$ value \\
\hline pNN50 (\%) & $0.13(0.55)$ & $5.36(8.99)$ & -4.47 & $<10^{-4}$ \\
pNN40 (\%) & $0.38(1.48)$ & $8.87(12.76)$ & -5.08 & $<10^{-5}$ \\
pNN30 (\%) & $1.05(3.45)$ & $14.78(17.17)$ & -6.03 & $<10^{-7}$ \\
pNN20 (\%) & $2.93(7.55)$ & $25.88(21.68)$ & -7.69 & $<10^{-10}$ \\
pNN10 (\%) & $9.48(15.08)$ & $48.90(22.91)$ & -11.10 & $<10^{-18}$ \\
\hline
\end{tabular}

Values are presented as mean (standard deviation). pNNx: the percentage of absolute differences in successive normal cardiac interbeat intervals greater than $\mathrm{x}$ msec 
of our knowledge, this is the first report to investigate the general $\mathrm{pNNx}$ HRV statistics in patients treated with psychotropic medication. The results of our study suggest that the pNNx statistics may be useful in evaluating the neuroautonomic dysfunction in patients treated with clozapine, complementing the traditionally computed pNN50 value.

The most significant separation between the two groups was observed at pNN5, indicating the importance of analyzing very small variations of interbeat interval in discriminating normal and pathological heart rate behavior patterns. ${ }^{16}$ Previous studies have shown that pNN50, which is a good index of parasympathetic cardiac modulation, was significantly diminished in schizophrenic patients. ${ }^{10,14,15}$ The conventional time domain HRV measures, such as RMSSD including pNN50 were estimated from the differences between successive interbeat intervals. Both RMSSD and pNN50 were reported to be useful in reflecting the fast alteration of parasympathetic activity and also evaluating the effects of various antipsychotics on autonomic neurocardiac function (ANF). ${ }^{10,15,24,26}$ Previous studies have shown that the HF fluctuation of RR intervals mainly reflects the vagal modulation, and that $\mathrm{HF}$, which is related to respiratory sinus arrhythmia (RSA), is another index to assess the fast parasympathetic modulation. ${ }^{18,27,28}$ In the present study, the pNNx values with $\mathrm{x} \leq 20 \mathrm{msec}$ were shown to correlate significantly well with RMSSD and with HF in the patient group, while the pNNx values with $x \geq 30$ msec showed most significant correlations with RMSSD and with HF in the control group. The difference of significant interclass correlations between the two groups supports previous reports that the pNN50 index were not sufficiently sensitive to detect the effects of certain antipsychotic or anticholinergic medication on ANF. ${ }^{29}$ According to our study, the pNNx values with $x \leq 20$ msec instead of the conventional $50 \mathrm{msec}$ might be useful candidates for the detection of subtle changes of parasympathetic and vagal activity in patients receiving various kinds of antipsychotic drugs. In addition, the parameters can be useful in HRV biofeedback measures to assess RSA activity. ${ }^{27,28,30}$

It should be noted that clozapine has potent anticholinergic and parasympatholytic effects. ${ }^{24}$ Evidence now exists for a strong association between reduced HRV and psychotropic drugs with anticholinergic properties includeing antipsychotics and tricyclic antidepressants. ${ }^{4,8-10,24,31}$ In addition, the effects of alpha adrenoceptor modulation on HRV via sympathetic and parasympathetic inputs were reported. ${ }^{19,32,33}$ Therefore, a significant reduction in pNNx statistics is assumed to be explicitly as-sociated with the direct effects of clozapine, although the psychotic symptom itself, might contribute to the decreased heart rate dynamics. Since the novel atypical anti- psychotics not only affect the dopaminergic receptors, but also exert significant effects on the serotonergic, cholinergic, and $\alpha$-adrenergic receptors, neurocardiac function integrity can be differentially affected by the distinct effects of these drugs on various receptors in the central and peripheral nervous systems. ${ }^{24}$ Further studies are warranted to examine the differential effects of the various atypical drugs on the general pNNx statistics.

The interpretation of the results of the present study should be considered in light of some limitations. Since the clozapine plasma level was not measured, we could not exclude the possibility that the plasma drug concentration might have influenced the pNNx values. Considering that the relationship between plasma antipsychotic drug level and heart rate dynamics may change depending on the phase of drug treatment, ${ }^{9}$ future studies should include measurement of plasma drug concentration. It is possible that the decreased heart rate dynamics observed in clozapine-treated subjects are associated with the psychotic symptom itself, as well as with the effects of the medication. ${ }^{10,34-36}$ Recent studies revealed cardiac autonomic dysfunction in patients with acute schizophrenia, which appears to be mainly related to reduced vagal and increased sympathetic modulation..$^{10,34-36}$ The crosssectional design of the study limits the firm interpretation of the results observed. Future prospective studies are required to evaluate the heart rate behavior in drug-naïve schizophrenic patients and to investigate the contribution of antipsychotic treatment to neuroautonomic modulation in these patients. ${ }^{37}$

In conclusion, the present study shows that $\mathrm{pNNx}$ with $\mathrm{x}<50$ msec provides more robust discrimination between the groups than the conventional pNN50 value, suggesting the importance of analyzing very small variations of interbeat interval in discriminating normal and pathological heart rate behavior patterns. The results imply that the general pNNx statistics may be applied and useful in evaluating the neuroautonomic dysfunction in patients treated with clozapine, complementing the traditionally computed pNN50 value. Subtle fluctuations in sinus rhythm quantified by pNNx statistics are thought to provide information about the very short term control of heart rate dynamics related to parasympathetic regulation. ${ }^{12,13,16}$ Novel analysis of HRV basing on the threshold of successive RR interval differences may serve as a useful noninvasive method providing a window into the alterations of complex interrelationships between brain and heart, and may have significant utility in clinical and research applications. $^{6}$

- Acknowledgments

For J-H Kim, this study was supported by the Choi Shin Hae Rese- 
arch Fund of Korean Neuropsychiatry Research Foundation in 2005. For S-H Yi, this work was supported by the Inje Research and Scholarship Foundation in 2005. This study was supported in part by the Brain Korea 21 project for Medicine, Dentistry, and Pharmacy (Seoul National University) and by Grant No. 04-2006-0540 from Seoul National University Hospital. This work was not funded by any pharmaceutical company, but the corresponding author has previously received grant, research support and/or honorarium from Norvatis, Janssen, Eli Lilly, Pfizer, Sanofi-aventis, Otsuka, Astra-zeneca, GlaxoSmithKline and Organon

\section{REFERENCES}

1. Huikuri HV, Kessler KM, Terracall E, Castellanos A, Linnaluoto MK, Myerburg RJ. Reproducibility and circadian rhythm of heart rate variability in healthy subjects. Am J Cardiol 1990;65:391-393.

2. Haapaniemi TH, Pursiainen V, Korpelainen JT, Huikuri HV, Sotaniemi KA, Myllylä VV. Ambulatory ECG and analysis of heart rate variability in Parkinson's disease. J Neurol Neurosurg Psychiatry 2001;70: 305-310.

3. Ansakorpi H, Korpelainen JT, Huikuri HV, Tolonen U, Myllylä VV, Isojärvi JI. Heart rate dynamics in refractory and well controlled temporal lobe epilepsy. J Neurol Neurosurg Psychiatry 2002;72:26-30.

4. Yeragani VK, Pesce V, Jayaraman A, Roose S. Major depression with ischemic heart disease: effects of paroxetine and nortriptyline on longterm heart rate variability measures. Biol Psychiatry 2002;52:418-429.

5. Kim W, Woo JM, Chae JH. Heart rate variability in psychiatry. J Korean Neuropsychiatr Assoc 2005;44:176-184.

6. Goldberger AL, Amaral LA, Hausdorff JM, Ivanov PCh, Peng CK, Stanley HE. Fractal dynamics in physiology: alterations with disease and aging. Proc Natl Acad Sci U S A 2002;99 Suppl 1:2466-2472.

7. Toichi M, Kubota Y, Murai T, Kamio Y, Sakihama M, Toriuchi T, et al The influence of psychotic states on the autonomic nervous system in schizophrenia. Int J Psychophysiol 1999;31:147-154.

8. Eschweiler GW, Bartels M, Längle G, Wild B, Gaertner I, Nickola M. Heart-rate variability (HRV) in the ECG trace of routine EEGs: fast monitoring for the anticholinergic effects of clozapine and olanzapine? Pharmacopsychiatry 2002;35:96-100.

9. Agelink MW, Sayar K, Klieser E. Usefulness of heart rate variability (HRV) for monitoring clozapine plasma levels. Pharmacopsychiatry 2003;36:166-167.

10. Kim JH, Yi SH, Yoo CS, Yang SA, Yoon SC, Lee KY, et al. Heart rate dynamics and their relationship to psychotic symptom severity in clozapine-treated schizophrenic subjects. Prog Neuropsychopharmacol Biol Psychiatry 2004;28:371-378.

11. Mujica-Parodi LR, Yeragani V, Malaspina D. Nonlinear complexity and spectral analyses of heart rate variability in medicated and unmedicated patients with schizophrenia. Neuropsychobiology 2005;51:10-15.

12. Ewing DJ, Neilson JM, Travis P. New method for assessing cardiac parasympathetic activity using 24 hour electrocardiograms. Br Heart J 1984;52:396-402.

13. Bigger JT Jr, Kleiger RE, Fleiss JL, Rolnitzky LM, Steinman RC, Miller JP. Components of heart rate variability measured during healing of acute myocardial infarction. Am J Cardiol 1988;61:208-215.

14. Malaspina D, Bruder G, Dalack GW, Storer S, Van Kammen M, Amador X, et al. Diminished cardiac vagal tone in schizophrenia: associations to brain laterality and age of onset. Biol Psychiatry 1997; 41:612-617.

15. Mueck-Weymann M, Rechlin T, Ehrengut F, Rauh R, Acker J, Dittmann RW, et al. Effects of olanzapine and clozapine upon pulse rate variability. Depress Anxiety 2002;16:93-99.

16. Mietus JE, Peng CK, Henry I, Goldsmith RL, Goldberger AL. The pNNx files: re-examining a widely used heart rate variability measure. Heart 2002;88:378-380.

17. Rechlin T, Claus D, Weis M. Heart rate variability in schizophrenic patients and changes of autonomic heart rate parameters during treatment with clozapine. Biol Psychiatry 1994;35:888-892.

18. Cohen H, Loewenthal U, Matar M, Kotler M. Association of autonomic dysfunction and clozapine. Heart rate variability and risk for sudden death in patients with schizophrenia on long-term psychotropic medication. Br J Psychiatry 2001;179:167-171.

19. Yeragani VK, Tancer M, Uhde T. Heart rate and QT interval variability: abnormal alpha-2 adrenergic function in patients with panic disorder. Psychiatry Res 2003;121:185-196.

20. American Psychiatric Association. Diagnostic and Statistical Manual of Mental Disorders. 4th ed. Washington DC, American Psychiatric Press, 1994, p.273-290.

21. Guy W. ECDEU assessment for psychopharmacology. Washington DC, HEW Public Health Service; 1976.

22. Hall RC. Global assessment of functioning. A modified scale. Psychosomatics 1995;36:267-275.

23. Kay SR, Fiszbein A, Opler LA. The positive and negative syndrome scale (PANSS) for schizophrenia. Schizophr Bull 1987;13:261-276.

24. Agelink MW, Majewski T, Wurthmann C, Lukas K, Ullrich H, Linka $\mathrm{T}$, et al. Effects of newer atypical antipsychotics on autonomic neurocardiac function: a comparison between amisulpride, olanzapine, sertindole, and clozapine. J Clin Psychopharmacol 2001;21:8-13.

25. Korpelainen JT, Sotaniemi KA, Huikuri HV, Myllylä VV. Abnormal heart rate variability as a manifestation of autonomic dysfunction in hemispheric brain infarction. Stroke 1996;27:2059-2063.

26. Kim JH, Yi SH, Park KT, Ahn YM, Lee KY, Yang SA, et al. Diminished neurocardiac dynamics associated with antipsychotic-induced extrapyramidal syndrome. Eur Neuropsychopharmacol 2006;16:459-463.

27. Löllgen D, Müeck-Weymann M, Beise RD. The deep breathing test: median-based expiration-inspiration difference is the measure of choice Muscle Nerve 2009;39:536-544

28. Mueck-Weymann M, Janshoff G, Mueck H. Stretching increases heart rate variability in healthy athletes complaining about limited muscular flexibility. Clin Auton Res 2004;14:15-18.

29. Malaspina D, Dalack G, Leitman D, Corcoran C, Amador XF, Yale S, et al. Low heart rate variability is not caused by typical neuroleptics in schizophrenia patients. CNS Spectr 2002;7:53-57.

30. Siepmann M, Aykac V, Unterdörfer J, Petrowski K, Mueck-Weymann M. A pilot study on the effects of heart rate variability biofeedback in patients with depression and in healthy subjects. Appl Psychophysiol Biofeedback 2008;33:195-201.

31. Cohen H, Loewenthal U, Matar MA, Kotler M. Reversal of pathologic cardiac parameters after transition from clozapine to olanzapine treatment: a case report. Clin Neuropharmacol 2001;24:106-108.

32. Duschek S, Heiss H, Werner N, Reyes del Paso GA. Modulations of autonomic cardiovascular control following acute alpha-adrenergic treatment in chronic hypotension. Hypertens Res 2009;32:938-943.

33. Tank J, Diedrich A, Szczech E, Luft FC, Jordan J. Alpha-2 adrenergic transmission and human baroreflex regulation. Hypertension 2004;43: 1035-1041.

34. Bär KJ, Wernich K, Boettger S, Cordes J, Boettger MK, Löffler S, et al. Relationship between cardiovagal modulation and psychotic state in patients with paranoid schizophrenia. Psychiatry Res 2008;157:255257.

35. Bär KJ, Boettger MK, Koschke M, Schulz S, Chokka P, Yeragani VK, et al. Non-linear complexity measures of heart rate variability in acute schizophrenia. Clin Neurophysiol 2007;118:2009-2015.

36. Bär KJ, Koschke M, Boettger MK, Berger S, Kabisch A, Sauer H, et al. Acute psychosis leads to increased QT variability in patients suffering from schizophrenia. Schizophr Res 2007;95:115-123.

37. Bär KJ, Koschke M, Berger S, Schulz S, Tancer M, Voss A, et al. Influence of olanzapine on QT variability and complexity measures of heart rate in patients with schizophrenia. J Clin Psychopharmacol 2008; 28:694-698. 